\title{
Rockefeller University
}

\section{Digital Commons@RU}

1990

\section{Kolmogorov Inertial Range for Inhomogeneous Turbulent Flows}

Bruce Knight

Lawrence Sirovich

Follow this and additional works at: http:/ / digitalcommons.rockefeller.edu/knight_laboratory

Part of the Life Sciences Commons

\section{Recommended Citation}

Knight, B. W., Jr. and Sirovich, L. (1990) Kolmogorov inertial range for inhomogeneous turbulent flows. Phys. Rev. Lett. 65: 1356-1359.

This Article is brought to you for free and open access by the Laboratories and Research at Digital Commons @ RU. It has been accepted for inclusion in Knight Laboratory by an authorized administrator of Digital Commons @ RU. For more information, please contact mcsweej@mail.rockefeller.edu. 


\title{
Kolmogorov Inertial Range for Inhomogeneous Turbulent Flows
}

\author{
B. Knight and L. Sirovich \\ Center for Fluid Mechanics, Turbulence and Computation, Brown University, Providence, Rhode Island 02912 \\ and Rockefeller University, New York, New York 1002I \\ (Received 16 April 1990)
}

\begin{abstract}
The Kolmogorov argument for the existence of an inertial range is reexamined in situations for which neither Fourier modes nor homogeneity and local isotropy are natural assumptions. Scaling arguments are shown which are still valid, and generalizations to the $-\frac{5}{3}$ law are given for the eigenvalue spectrum of the two-point velocity-correlation matrix. Results from several different numerical simulations are presented. Data derived from simulations of channel and convection flows show that a sensible inertial range appears at very modest Reynolds numbers.
\end{abstract}

PACS numbers: $47.25 .-\mathrm{c}$

The idea of the inertial subrange ${ }^{1,2}$ has had a profound effect on research in turbulence (see Refs. 3-5 for reviews). In particular, a number of experimental investigations have demonstrated the presence of the famous $-\frac{5}{3}$ power law of the Kolmogorov spatial-energy spectrum over relatively large ranges of wave numbers, when the Reynolds number (Re) of the flow is large. ${ }^{6-10}$ (In all instances, only one-dimensional spectra have been assayed.) The universal features of the inertial range also lie at the heart of most theoretical attempts to reduce the turbulent form of the Navier-Stokes (NS) equations. ${ }^{11-14}$

The Kolmogorov spectrum may be approached through dimensional reasoning. In particular, if one considers the energy density spectrum of the velocity

$$
e=\frac{1}{V} \int \mathbf{u}^{2} d \mathbf{x}=\int \mathscr{E}(\mathbf{k}) d \mathbf{k},
$$

then, since the dimension of $e$ is velocity squared, $\operatorname{dim}[e]=l^{2} / t^{2}$,

$$
\operatorname{dim}[\mathscr{E}]=l^{5} / t^{2} \text {. }
$$

Next, one defines the dissipation rate, $\epsilon=U^{3} / L$, where $U$ is a characteristic velocity and $L$ a characteristic length. Then since the kinematic viscosity $v$ is such that $\operatorname{dim}[v]=l^{2} / t$, it follows that the Kolmogorov length scale is

$$
\eta=\left(v^{3} / \epsilon\right)^{1 / 4}=1 / \kappa .
$$

It is then argued that for Reynolds number $\mathrm{Re}$ $=U L / v \uparrow \infty$, and $k \gg L^{-1}$, the spectrum should take on a universal form independent of the driving mechanisms and dependent only upon the energy transfer rate $\epsilon$; dimensional reasoning then yields

$$
\mathscr{E} k^{11 / 3} / \epsilon^{2 / 3}=f(\mathbf{k} / \kappa) \text {. }
$$

With the added assumption of local isotropy,

$$
\mathscr{E}=E(k) / 4 \pi k^{2},
$$

where $E(k)$ is the energy density per unit scalar wave number on a sphere with radius of wave number $k$, so that the universal form is given by

$$
E(k) k^{5 / 3} / \epsilon^{2 / 3}=F(k / \kappa) .
$$

The inertial range is obtained by assuming in addition,

$$
L^{-1} \ll k \ll \kappa
$$

from which it follows that

$$
E \propto \epsilon^{2 / 3} k^{-5 / 3},
$$

or equivalently for energy per vector wave number,

$$
\mathscr{E} \propto \epsilon^{2 / 3} k^{-11 / 3} .
$$

Landau and Lifshitz ${ }^{15}$ have suggested that intermittency would modify (8). For this reason Kolmogorov ${ }^{2}$ proposed a log-normal model for dealing with intermittency, and Mandelbrot ${ }^{16,17}$ has shown that intermittency might lead to a steeper falloff than (11) below (see also the article by Frisch ${ }^{18}$ ). One may show this does not conflict with the arguments of dimensional reasoning and since this plays no essential role here we leave this point without further discussion.

The derivation given above assumes a Fourier spectral representation which is justified only for turbulence which is statistically stationary under translations in all directions. Following (5) above, the further assumption of isotropy under any rotation at any point is made as well. Here we extend the Kolmogorov argument to the much commoner situations for which full translational invariance and isotropy are not applicable or natural. Instead, we use the empirical eigenfunction approach as a framework for this development, ${ }^{19-24}$ since these eigenfunctions constitute an intrinsically defined and optimal basis set. This we now discuss briefly.

We continue to consider turbulent flows which are statistically stationary in time and denote the velocity by $\mathbf{v}=\left(v_{1}, v_{2}, v_{3}\right)$, and by

$$
\left\{\mathbf{v}^{(n)}(\mathbf{x})\right\}
$$

an ensemble of states on the turbulent attractor [say $\mathbf{v}^{(n)}=\mathbf{v}\left(\mathbf{x}, t_{n}\right)$ where the flow is sampled at uniform times]. We suppose that the mean flow has been subtracted, so that

$$
\langle\mathbf{v}\rangle=0 \text {, }
$$

where the brackets denote an ensemble average. The two-point correlation matrix is defined by the outer prod- 
uct of the velocity fields,

$$
\mathbf{K}\left(\mathbf{x}, \mathbf{x}^{\prime}\right)=\left\langle\mathbf{v}(\mathbf{x}) \otimes \mathbf{v}\left(\mathbf{x}^{\prime}\right)\right\rangle
$$

and from this the empirical (vector) eigenfunctions are then given by

$$
\int \mathbf{K}\left(\mathbf{x}, \mathbf{x}^{\prime}\right) \phi^{(n)}\left(x^{\prime}\right) d x^{\prime}=\lambda_{n} \phi^{(n)}(\mathbf{x})
$$

The kernel $\mathbf{K}$ is a Hermitian, non-negative operator, and under mild assumptions, the empirical eigenfunctions can be shown to form a complete orthonormal set. Furthermore, in a well-defined sense this set, ordered by declining eigenvalues and truncated at any point, is the optimal partial basis set by which to represent a flow. It follows from these properties that the energy is given by

$$
\hat{e}=\left\langle\int v_{i}(\mathbf{x}) v_{i}(\mathbf{x}) d \mathbf{x}\right\rangle=\operatorname{Tr} \mathbf{K}=\sum_{n} \lambda_{n},
$$

with

$$
\operatorname{dim}[\hat{e}]=\operatorname{dim}\left[\lambda_{n}\right]=l^{5} / t^{2}
$$

An eigenvalue $\lambda_{n}$ represents the average energy allocated to the projection of the velocity field along the corresponding mode $\phi^{(n)}$. Thus the summation in (14) represents the natural generalization from homogeneous turbulence, of the energy integral in Fourier space, (1). Each $\lambda_{n}$ represents the energy in the corresponding state, and is a generalization of $\mathscr{E}(\mathbf{k})$ and carries the same physical dimensions.

Next, we apply dimensional reasoning to the generalized energy spectrum for two computational simulations for which sufficient data exist to explore the results.

Channel flow (Refs. 25-27). - We consider plane Poiseuille flow in the $x$ direction, of infinite extent in the spanwise $y$ direction, and bounded in the vertical direction by parallel planes spaced a distance $L$ apart. Because $x$ and $y$ are homogeneous directions an empirical eigenfunction has the form

$$
\phi^{(q)}=\Phi^{(q)}\left(z ; k_{x}, k_{y}\right) \exp \left(i k_{x} x+i k_{y} y\right),
$$

and hence

$$
\lambda=\lambda^{(q)}\left(k_{x}, k_{y} ; U, L, v\right) .
$$

Here $q$ represents the "vertical quantum number" of the correlation operator, and we have introduced the parameters of the problem.

If we apply to (17) the dimensional reasoning used above to obtain (4), we obtain for $k \gg L^{-1}$ and for $\operatorname{Re} \uparrow \infty$ that

$$
\frac{\lambda^{(q)}}{\epsilon^{2 / 3} k_{\perp}^{-11 / 3}}=f_{q}\left(\frac{k_{\perp}}{\kappa}, \frac{k_{x}}{k_{y}}\right)
$$

with

$$
k_{\perp}=\left(k_{x}^{2}+k_{y}^{2}\right)^{1 / 2} .
$$

As before, if it is further assumed that $k_{\perp} \ll \kappa$, then

$$
\lambda^{(q)} \sim \epsilon^{2 / 3} k_{\perp}^{-11 / 3} f_{q}\left(k_{x} / k_{y}\right) .
$$

It should be noted that no assumption of local isotropy has been made. Nor does (20) imply that modes with different $Q$ are decoupled from energy exchange with each other, which observation becomes clear when we note that isotropic turbulence with Kolmogorov spectrum yields a particular case of (20): we may freely choose a direction as the vertical $z$ direction and (16) still holds with $\Phi^{(q)}=\exp (i q z / L)$ if we quantize our Fourier modes within a box whose vertical dimension is $L / 2 \pi$. If we hold $q$ fixed while $k_{\perp}$ becomes large, then $k \rightarrow k_{\perp}$ in (12) which then becomes a special case of (20) with $f_{q}\left(k_{z} / k_{y}\right)=$ const. Before illustrating and commenting on (20) we consider one more case.

Rayleigh-Bénard (RB) convection (Refs. 28 and 29). - We consider RB convection between parallel planes at $z=0$ and $L$ and which are held at temperatures $T_{0}+\Delta T$ and $T_{0}-\Delta T$, respectively. The flow is taken to be unbounded in the $x$ and $y$ directions. An additional physical parameter, the Prandtl number Pr (ratio of thermal conductivity to kinematic viscosity), enters this problem. Again the empirical eigenfunctions take on the product form (16). The eigenvalues now have the form

$$
\lambda^{(q)} / \epsilon^{2 / 3} k_{\perp}^{1 / 3}=f_{q}\left(k_{\perp} / \kappa ; \operatorname{Pr}\right),
$$

since there is no preferred horizontal direction. Therefore in the Kolmogorov limit we have instead of (20),

$$
\lambda^{(q)} \sim \epsilon^{2 / 3} k_{\perp}^{-11 / 3} f_{q}(\mathrm{Pr}) .
$$

In Figs. 1-3 we plot the results of simulations of channel flow $^{25-27}$ and RB convection. ${ }^{28,29}$ Each of these cases has a high degree of symmetry and the question of treating degeneracies arises. If we note that a slight perturbation in geometry introduces a break in the degeneracy, then it is seen that in allocating energy to individual modes it would be inappropriate to multiply a degenerate eigenvalue by its degeneracy. Such eigenvalues rather should be repeated, or infinitesimally displaced a number

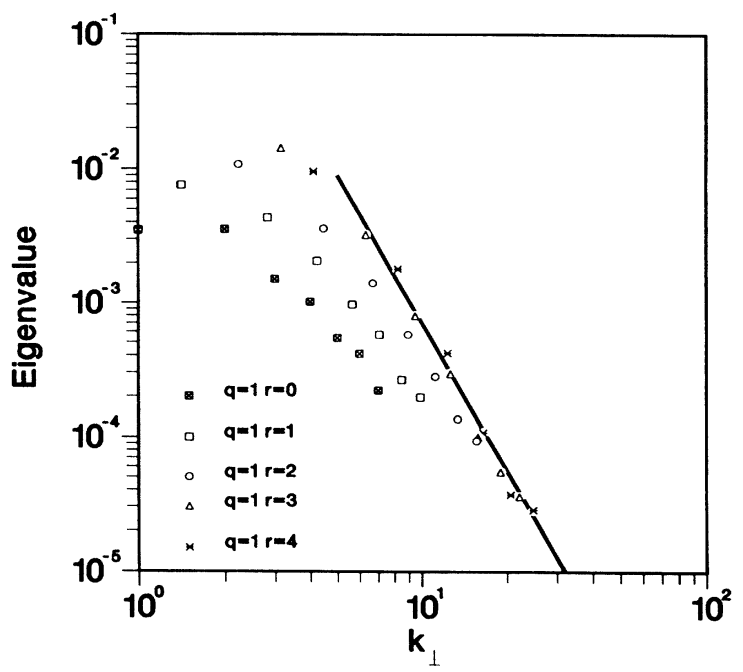

FIG. 1. $\lambda_{n}$ vs the horizontal wave number $k_{\perp}=\left(k_{x}^{2}+k_{y}^{2}\right)^{1 / 2}$ for the indicated values of orientation, $r=k_{y} / k_{x}$, and quantum number, $q$. Data based on channel calculations of Handler and co-workers (Refs. 25 and 26). (64 grid points in the vertical.) Solid line corresponds to (24). 


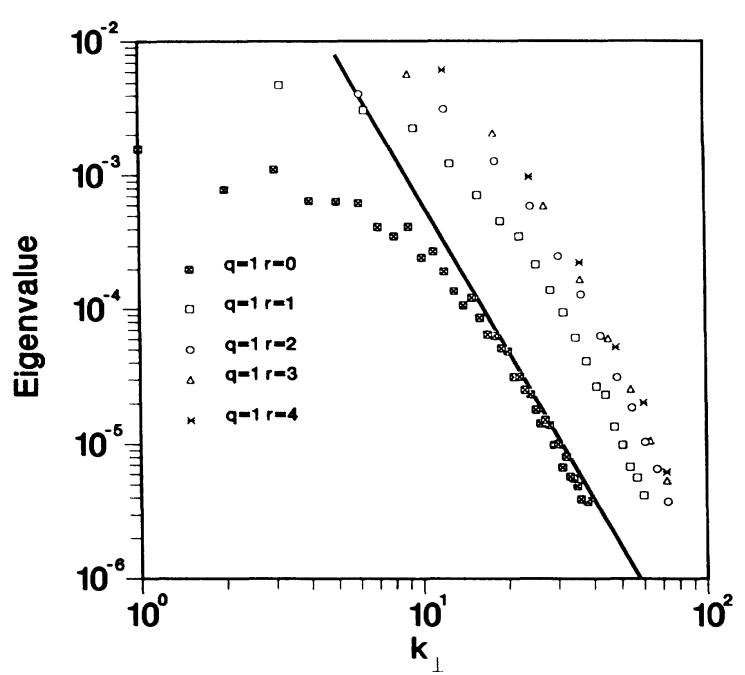

FIG. 2. Same as in Fig. 1. Data based on channel calculation of Kim, Moin, and Moser (Ref. 27). (129 grid points in the vertical.)

of times appropriate to account for the degeneracy.

The cases shown in Fig. 1 come from Handler, Hendricks, and Leighton ${ }^{25}$ and Handler ${ }^{26}$ and correspond to channel flow at $R e=2128$, based on half-channel-width. This Reynolds number is only weakly turbulent. Here only the first quantum number is considered and a variety of orientations, $r=\left(k_{x} / k_{y}\right)^{-1}$, shown [see (20)]. Although this is a numerically well-resolved flow, relatively few points are seen on each plot since a separate plot is necessary for each orientation, $r$. Even at this quite low Reynolds number, a modest inertial range is evidenced by the $-\frac{11}{3}$ slope which the figure shows. The comparable one-dimensional Fourier spectra in Ref. 25 show no inertial range and are of questionable interpretation because this turbulence is strongly inhomogeneous. Figure 2 derives from the simulation of Kim, Moin, and Moser $^{27}$ for a similar geometry but the substantially higher (though low) Reynolds number of $\mathrm{Re}=3300$. A more pronounced inertial range may be seen for modes with wave number at several angles to the mean flow and with first quantum number.

In Fig. 3 we show plots from a Bénard convection simulation ${ }^{28,29}$ for a Rayleigh number of $6.4 \times 10^{6}$ (and nominal Reynolds number $\approx 10^{3}$ ). In this instance we observe a substantial inertial range. To account for finer details of the figure, we may argue that the effective mode Reynolds number must decrease as the quantum number increases, in consequence of more closely spaced zero crossings. This would naturally lead to less energy in modes with higher $q$, as the figure shows; and because it starts at lower energy, a sequence of modes at a higher fixed $q$ shows a shorter inertial range.

The dynamical signature of an infinite inertial range is a mode-number-independent rate of energy cascade, from low to high mode numbers, that is local in mode-

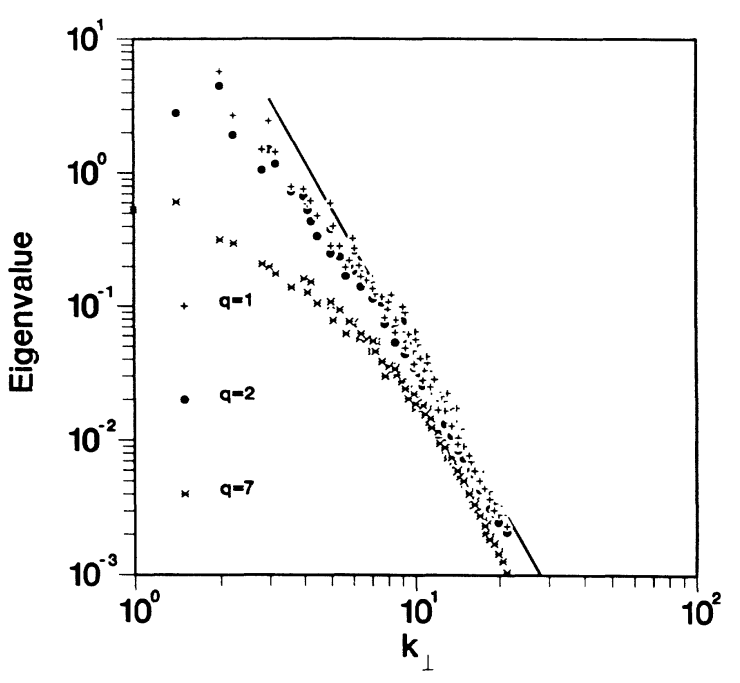

FIG. 3. $\lambda_{n}$ vs $k_{\perp}$ for the Rayleigh-Bénard computation of Refs. 28 and 29. (97 grid points in the vertical.) Solid line corresponds to (26).

number space. ${ }^{30-32}$ We have not determined to what degree a constant energy cascade accompanies the finite, approximate power-law ranges presented in this Letter.

The arguments above made use of the fact that the flow had homogeneous directions and hence that a Fourier decomposition is natural in those directions. This still leaves open the question of an inertial range if no homogeneous directions are present. We may generalize our result with a heuristic argument.

In the special case of homogeneous turbulence, in the inertial range Eq. (9) states that the average energy of a single sinusoidal mode at wave number $k$ scales as $\mathscr{E} \propto k^{-11 / 3}$. But the Fourier modes that have mode energies larger than the energy found at wave number $k$ are those with smaller $k$, which may be counted in number as

$$
N \propto k^{3}, \text { whence } k \propto N^{1 / 3} .
$$

Therefore, in the case of homogeneous turbulence, when $k$ is substituted out in favor of $N$,

$$
\lambda_{N} \propto N^{-11 / 9} .
$$

Theoretical arguments can be advanced which suggest that this result may be universal. In particular, as index increases, with some generality eigenfunctions become locally sinusoidal in regions where they show large amplitudes. In Fig. 4 we present $\lambda_{N}$ of Eq. (13) versus $N$ for four large-scale simulations. In each case the dashed line corresponds to the $-\frac{11}{9}$ power law of (24). We must remember that if context makes wave numbers appropriate, then the extent of an inertial range in equivalent wave number (measured in decades) is shorter than that shown here, by a factor of 3 according to Eq. (23).

Figure 4, curve $a$, derives from the channel flow simu- 


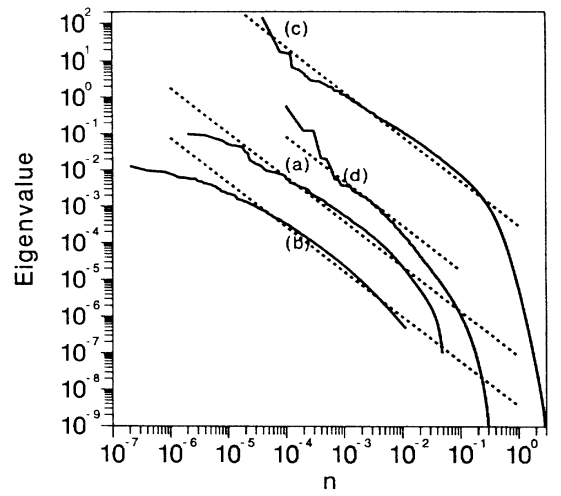

FIG. 4. $\lambda_{n}$ vs index $n$ for (curve $\left.a\right)$ channel flow $(\operatorname{Re}=2128)$ (Refs. 25 and 26 ), (curve $b$ ) channel flow $(\operatorname{Re}=3300$ ) (Ref. $27)$, (curve $c$ ) Rayleigh-Bénard convection $\left(\mathrm{Ra} \approx 6.4 \times 10^{6}\right)$ (Ref. 28 and 29), (curve d) Rayleigh-Bénard convection $(\mathrm{Ra} \approx 46,000)$. Dashed lines correspond to (29) (Refs. 33 and 34). The plots have been displaced for purposes of illustration.

lation of Handler at half-width Reynolds number 2300 discussed above; similarly curve $b$ derives from Kim, Moin, and Moser $\mathrm{Re}=3300$ simulation (offset down by one decade). Curve $c$ is from the Bénard convection simulation at Rayleigh number $\mathrm{Ra}=6.4 \times 10^{6}$. Shown in the present way, this well-developed turbulence reveals an extensive inertial range. Curve $d$ is a Bénard convection simulation at the modest Rayleigh number of $\mathrm{Ra}=4.7 \times 10^{3},{ }^{33,34}$ for convection whose flow is constrained within a rectangular solid with square base. In this geometry there is no remaining translational invariance, and the wave-number concept is inapplicable to the flow eigenfunctions; again the energy eigenvalues show the $-\frac{11}{9}$ power scaling across a short but evident inertial range.

We gratefully acknowledge our debt to K. Ball, S. Balachandar, R. Handler, R. Moser, and H. Park, who furnished us with the data presented here, and to $R$. Kraichnan and V. Yakhot for helpful comments. This work was supported by U.S. Defense Advanced Research Projects Agency under Contract No. N00014-86-K0754.

${ }^{1}$ A. N. Kolmogorov, C. R. Acad. Sci U.S.S.R. 30, 301-538 (1941).

${ }^{2}$ A. N. Kolmogorov, J. Fluid Mech. 13, 82 (1962).

${ }^{3}$ A. S. Monin and A. M. Yaglom, Statistical Fluid Mechanics, (MIT Press, Cambridge, 1975), Vol. 2.

${ }^{4} \mathrm{G}$. K. Batchelor, Theory of Homogeneous Turbulence (Cambridge Univ. Press, Cambridge, 1953).

${ }^{5} \mathrm{H}$. Tennekes and J. L. Lumley, A First Course in Turbulence (MIT Press, Cambridge, 1972).
${ }^{6}$ H. L. Grant, R. W. Stewart, and A. Moilliet, J. Fluid Mech. 12, 241 (1962).

${ }^{7}$ J. Laufer, NACA Report No. 1174, 1954 (unpublished).

${ }^{8}$ S. Pond, S. D. Smith, P. F. Hamblin, and R. W. Burling, J. Atom. Sci. 23, 376 (1966).

${ }^{9} \mathrm{C}$. Meneveau and K. R. Sreenivasan (to be published).

${ }^{10}$ A. S. Gurich, B. M. Kopvou, L. R. Tsvang, and A. M. Yaglom, in Atmospheric Turbulence and Radio Wave Propagation, edited by A. M. Yaglom and V. I. Tatarski (Nauka, Moscow, 1967), p. 30.

${ }^{11}$ D. C. Leslie, Developments in the Theory of Turbulence (Clarendon, Oxford, 1973).

${ }^{12}$ S. A. Orszag, in Fluid Dynamics, Proceedings of the 1973 Les Houches Summer School, edited by R. Balian and J. L. Penbe (Gordon and Breach, New York, 1977).

${ }^{13}$ M. Lesieur, Turbulence in Fluids (Martinus Nijhoff, The Hague, 1987).

14J. O. Hinze, Turbulence (McGraw-Hill, New York, 1975), 2nd ed.

${ }^{15}$ L. D. Landau and E. M. Lifshitz, Course of Theoretical Physics, Fluid Mechanics Vol. 6 (Pergamon, New York, 1959).

${ }^{16}$ B. B. Mandelbrot, J. Fluid Mech. 62, 331 (1974).

${ }^{17}$ B. B. Mandelbrot, in Turbulence and the Navier-Stokes Equation, edited by R. Temam, Lecture Notes in Mathematics (Springer-Verlag, Berlin, 1976), Vol. 565, p. 121.

${ }^{18} \mathrm{U}$. Frisch, in Nonlinear Dynamics, edited by R. Helleman [N.Y. Acad. Sci. 357, 359 (1980)].

${ }^{19} \mathrm{~J}$. L. Lumley, in Transition and Turbulence, edited by $\mathrm{R}$. E. Meyer, 242 (Academic, New York, 1981), pp. 215-242.

${ }^{20} \mathrm{~J}$. L. Lumley, Stochastic Tools in Turbulence (Academic, New York, 1970).

${ }^{21}$ J. L. Lumley, in Atmospheric Turbulence and Radio Wave Propagation, edited by A. M. Yaglom and V. I. Tatarski (Nauka, Moscow, 1967), pp. 116-178.

${ }^{22}$ L. Sirovich, Q. Appl. Math. 45, 561 (1987); 45, 573 (1987); 45, 583 (1987).

${ }^{23}$ L. Sirovich, Physica (Amsterdam) 37D, 126-145 (1989).

${ }^{24}$ L. Sirovich and C. H. Sirovich, in Contemporary Mathematics, edited by Basil Nicolaenko (American Mathematical Society, Providence, 1989), Vol. 99, p. 277.

${ }^{25}$ R. Handler, E. W. Hendricks, and R. I. Leighton, NRL Memorandum Report No. 6410, 1989 (to be published).

${ }^{26}$ R. A. Handler, NRL Memorandum Report (to be published).

${ }^{27}$ J. Kim, P. Moin, and R. Moser, J. Fluid Mech. 177, 133 (1987).

${ }^{28}$ L. Sirovich, S. Balachandar, and M. R. Maxey, Phys. Fluids A 1, 1911 (1989).

${ }^{29}$ S. Balachandar, M. R. Maxey, and L. Sirovich, J. Sci. Comput. 4, 219 (1989).

${ }^{30}$ R. H. Kraichnan, Phys. Fluid 11, 265 (1968).

${ }^{31}$ R. H. Kraichnan, J. Atmos. Sci. 33, 1521 (1976).

${ }^{32}$ R. H. Kraichnan, Phys. Fluids 9, 1728 (1965).

${ }^{33}$ L. Sirovich and H. Park, Phys. Fluids. A 2, 1649 (1990).

${ }^{34}$ H. Park and L. Sirovich, Phys. Fluids. A 2, 1659 (1990). 